\title{
Identification of key pathways and genes shared between Crohn's disease and breast cancer using bioinformatics analysis
}

\author{
JIANG $\mathrm{ZHOU}^{1-3}$ and RONGCUN YANG ${ }^{1-3}$ \\ ${ }^{1}$ State Key Laboratory of Medicinal Chemical Biology; ${ }^{2}$ Key Laboratory of Bioactive Materials of The Ministry of Education; \\ ${ }^{3}$ Department of Immunology, School of Medicine, Nankai University, Tianjin 300071, P.R. China
}

Received November 18, 2018; Accepted July 8, 2020

DOI: $10.3892 /$ ol.2020.11981

\begin{abstract}
Patients with Crohn's disease (CD) have a high risk of developing breast cancer, suggesting that there may be shared molecular mechanisms underlying CD and breast cancer. The purpose of the present study was to identify the critical genes and pathways underlying these molecular similarities using bioinformatics analysis. Publicly available microarray expression data from the Gene Expression Omnibus were analyzed, and a total of 53 overlapping differentially expressed genes (DEGs) between the CD (vs. controls) and breast cancer (vs. controls) groups were identified. These common DEGs were then subjected to Gene Ontology and Kyoto Encyclopedia of Genes and Genomes pathway enrichment analyses. Subsequently, a gene interaction network of the DEGs was constructed using Cytoscape software, with its plug-in cytoHubba and Molecular Complex Detection. The gene interaction network and module analysis demonstrated that prostaglandin $\mathrm{G} / \mathrm{H}$ synthase 2 , interleukin $(I L) 1 \beta$ and CXCL8 were the major hub genes in the upregulated overlapping DEGs. The upregulated overlapping DEGs are found to be enriched in both the IL-17 and NF-kB signaling pathways. Taken together, the critical pathways and genes identified in the present study may help improve our understanding of why and how CD may contribute to the development of breast cancer.
\end{abstract}

\section{Introduction}

Inflammatory bowel disease (IBD), including ulcerative colitis and Crohn's disease (CD), is characterized by long-term relapsing inflammatory conditions in the gut (1). CD is associated with an increased risk of general cancer, such as colorectal cancer (2). Recent evidence suggests that the

Correspondence to: Professor Rongcun Yang, Department of Immunology, School of Medicine, Nankai University, 94 Weijin Road, Nankai, Tianjin 300071, P.R. China

E-mail: ryang@nankai.edu.cn

Key words: Crohn's disease, breast cancer, microarray, differentially expressed genes, functional enrichment analysis, gene interaction network inflammation in $\mathrm{CD}$, as a comorbidity, may contribute to the initiation of breast cancer (2). It has been agreed that $\mathrm{CD}$ is an important risk factor for the development of breast cancer (3). However, research on the impact of CD on the development of breast cancer is limited, particularly regarding the underlying molecular mechanisms.

A current study suggests that certain common genetic factors may exist that account for the association between Crohn's disease and breast cancer (4). Hence, the molecular mechanisms shared between CD and breast cancer should be investigated. To do this, the present study compared the differentially expressed genes (DEGs) in the peripheral blood cells of different cohorts, namely patients with CD vs. healthy controls, and patients with breast cancer vs. healthy controls. The overlapping genes and pathways were further studied by using bioinformatics tools, such as the Kyoto Encyclopedia of Genes and Genomes (KEGG) database. The present study aimed to analyze gene expression profiles and related pathways in the peripheral blood cells of patients with CD and breast cancer. Understanding the aberrant expression of the genes involved in these pathways may contribute to developing treatment for breast cancer in patients with CD.

\section{Materials and methods}

Datasets selection and preprocessing. To search for gene signatures shared by $\mathrm{CD}$ and breast cancer in the peripheral blood of women, the terms 'PBMC and Crohn's disease' or 'breast cancer' were used to search for datasets in Gene Expression Omnibus (5) and Array Express (https://www. ebi.ac.uk/arrayexpress). The dataset selection criteria were as follows: i) Preference for microarray datasets; ii) samples using peripheral blood mononuclear cells (PBMCs); iii) the number of samples was $>15$ and iv) processed data were eligible. According to these selection criteria, only two datasets (GSE3365 and GSE27562) (6,7) were identified. GSE3365 contained samples from 42 healthy controls and 59 patients with CD. The GSE27562 blood samples were collected from 57 women with breast cancer and 31 healthy women. Normalized datasets were downloaded from the GEO database (https://www.ncbi. nlm.nih.gov/gds). Datasets GSE3365 (Crohn's disease) and GSE27562 (breast cancer) were annotated according to the Affymetrix Human Genome U133A (https://www.ncbi.nlm. nih.gov/ geo/query/acc.cgi?acc=GPL96) and U133 Plus 2.0 
Array platforms (https://www.ncbi.nlm.nih.gov/geo/query/acc. cgi?acc $=$ GPL570) respectively. Probes were transformed to gene symbols. If the same gene symbol was mapped by multiple probes, their maximal value was regarded as the gene expression value. For the GSE3365 dataset, log conversion and quantile normalization were applied. Normalized datasets were subjected to principal component analysis (PCA), an orthogonal linear transformation can convert the related variables into irrelative variables (8). Samples that were clustered and/or not separated were discarded.

DEGs analysis. The limma package (version 3.31.22) supplied by Bioconductor was applied to analyze DEGs in CD or breast cancer PBMCs compared with normal samples. The list of the DEGs (in the form of a table) was obtained when the limma top table function was used. In this table, DEGs were identified according to the following criteria: i) ' $|F o l d-c h a n g e(F C)|>$ mean of $\log \mathrm{FC}$ of results table $+2 \mathrm{x}$ standard derivation of $\log \mathrm{FC}$ of results table' and ii) ' $\mathrm{P}<0.05$ '. To obtain overlapping DEGs between the two individual comparisons (CD vs. healthy controls and breast cancer vs. healthy controls), a Venn diagram was constructed to identify the overlapping DEGs in these groups. Then the overlapping DEGs were used in the following analyses.

Pathway enrichment analysis. To determine the function of identified overlapping DEGs, ClusterProfiler (http://bioconductor.org/packages/release/bioc/html/ clusterProfiler.html) with a series of strict cut-off (a variable value that can distinguish positive from negative results) was used to perform Gene Ontology (GO) and KEGG pathway analysis (9). GO analysis includes three categories: Biological process (BP); molecular function (MF) and cellular component (CC). For all GO term annotation and KEGG pathway enrichment analysis, false discovery rate $<0.05$ was selected as the level of significant difference. Gene abbreviations are defined in Table SI.

Gene interaction network (GI) analysis. The overlapping DEGs were selected after pathway enrichment analysis. Then these DEGs were submitted to the Search Tool for the Retrieval of Interacting Genes (STRING) database (http:/www.string-db. org/) to construct a protein-protein interaction network. With a combined score $>0.4$, a chart indicating the interactions of DEGs was exported for downstream analysis using Cytoscape software (version 3.4.0) (10). Significant modules were selected according to the default selection criteria (namely, Molecular Complex Detection scores $>2$ ). Next, the Cytoscape plugin cytoHubba was used to define hub genes of the DEGs interaction network with a degree topological analysis.

Pathway analysis. The 'pathview' package in Bioconductor was used to display the results, which included expression profiles of the corresponding genes (11). This tool links gene expression, gene regulations and related functions to pathways based on the KEGG analyses. Gene abbreviations are defined in Tables SII-IV.

\section{Results}

Overlapping DEGs. Using limma tools to analyze the GSE3365 and GSE27562 datasets, DEGs between CD (vs. healthy controls) and breast cancer (vs. healthy controls) were identified. In total, 606 DEGs, including 229 upregulated DEGs (Fig. 1A) and 377 downregulated DEGs (Fig. 1B) were identified between the CD vs. healthy controls (CD group), and 984 DEGs, including 575 upregulated DEGs (Fig. 1A) and 409 downregulated DEGs (Fig. 1B) were identified in patients with breast cancer vs. healthy controls (BC group). For the upregulated DEGs, there were 46 overlapping genes between the $\mathrm{BC}$ and $\mathrm{CD}$ groups (Fig. 1A and Table I); whereas there were seven overlapping downregulated genes (Fig. 1B and Table I). The results indicated that there existed overlapping DEGs between the $\mathrm{BC}$ and $\mathrm{CD}$ groups.

GO term annotation and KEGG pathway enrichment analysis. Functional annotation and pathway analyses of overlapping genes between the DEGs from the BC and CD groups were performed using the ClusterProfiler tool. The upregulated overlapping DEGs were primarily annotated with the GO terms 'cell chemotaxis', 'positive regulation of response to external stimulus' and 'response to lipopolysaccharide' in terms of BP, and 'cytokine activity', 'receptor ligand activity' and 'receptor regulator activity' in terms of MF (Table II). The downregulated overlapping DEGs were primarily annotated with the GO terms under the category of MF, such as 'palmitoyl-(protein) hydrolase activity' (Table II). The upregulated overlapping DEGs were primarily enriched in the following KEGG signaling pathways: 'IL-17, 'C-type lectin receptor, 'tumor necrosis factor (TNF) $\alpha$, 'NF- $\kappa \mathrm{B}$ ' and 'NOD-like receptor' (Fig. 2A). The downregulated overlapping DEGs were primarily enriched in the pathway of 'fatty acid elongation' (Fig. 2B). The gene functional enrichment analysis of theses DEGs suggested that they were mainly involved in inflammatory pathways.

Hub genes and significant modules identified from the GI network. Functional overlapping DEGs were submitted to the STRING database. For overlapping DEGs between the CD vs. BC group, network states showed 30 nodes and 67 edges. Based on this network, cytoscape software was applied to construct the GI network (Fig. 3A). Among the overlapping DEGs, ten hub DEGs according to the node degree were identified (Fig. 3B; Table III). Furthermore, significant modules that met the default selection criteria were obtained (Fig. 3C). For the intersecting DEGs between the CD vs. BC groups, the CXCL8, $I L 1 \beta$ and prostaglandin $\mathrm{G} / \mathrm{H}$ synthase 2 (PTGS2) genes were identified in the module (Table III). Based on the analysis of the hub gene and significant module, the interactions between the DEGs were identified.

Regulation of overlapping DEGs in enriched pathways. Several studies have shown that IL-17, C-type lectin receptor and $\mathrm{NF}-\kappa \mathrm{B}$ signaling pathways serve an important role in both $\mathrm{CD}$ and breast cancer pathology (12-16). Thus, the regulation and potential function of the overlapping DEGs between the $\mathrm{BC}$ and $\mathrm{CD}$ groups were analyzed in these enriched pathways in the BC group (Figs. 4-6). Indeed, similar pathway patterns were also identified in the CD group (data not shown). In Fig. 4, a number of upstream genes induced the degradation of inhibitor of $N F-\kappa B(I \kappa B)$, thereby allowing NF- $\kappa$ B translocation into the nucleus to activate inflammatory gene expression 
Table I. Genes commonly differentially expressed between Crohn's disease and breast cancer.

\begin{tabular}{|c|c|c|}
\hline Gene symbol & Gene name & Upregulated or downregulated \\
\hline AREG & Amphiregulin & Up \\
\hline BAG4 & BCL2 associated athanogene 4 & Up \\
\hline BCL3 & BCL3 transcription coactivator & $\mathrm{Up}$ \\
\hline C5AR1 & Complement $\mathrm{C} 5$ a receptor 1 & Up \\
\hline CCR1 & $\mathrm{C}-\mathrm{C}$ motif chemokine receptor 1 & Up \\
\hline CCRL2 & C-C motif chemokine receptor like 2 & Up \\
\hline CD83 & Cluster of differentiation 83 molecule & Up \\
\hline CD9 & Cluster of differentiation 9 molecule & Up \\
\hline CXCL2 & $\mathrm{C}-\mathrm{X}-\mathrm{C}$ motif chemokine ligand 2 & Up \\
\hline CXCL8 & $\mathrm{C}-\mathrm{X}-\mathrm{C}$ motif chemokine ligand 8 & Up \\
\hline DNAJC3 & DnaJ heat shock protein family (Hsp40) member C3 & Up \\
\hline DSC2 & Desmocollin 2 & Up \\
\hline DUSP5 & Dual specificity phosphatase 5 & Up \\
\hline EGR1 & Early growth response 1 & Up \\
\hline EGR2 & Early growth response 2 & Up \\
\hline EGR3 & Early growth response 3 & Up \\
\hline EPB41L3 & Erythrocyte membrane protein band 4.1 like 3 & Up \\
\hline FOSB & FosB proto-oncogene, AP-1 transcription factor subunit & Up \\
\hline FOSL2 & FOS like 2, AP-1 transcription factor subunit & Up \\
\hline G0S2 & G0/G1 switch 2 & Up \\
\hline GAB2 & GRB2 associated binding protein 2 & Up \\
\hline GABARAPL1 & GABA type A receptor associated protein like 1 & Up \\
\hline HBEGF & Heparin binding EGF like growth factor & Up \\
\hline IER3 & Immediate early response 3 & Up \\
\hline IL1B & Interleukin 1 beta & Up \\
\hline MAFB & MAF bZIP transcription factor B & Up \\
\hline MARCKS & Myristoylated alanine rich protein kinase $\mathrm{C}$ substrate & Up \\
\hline NAMPT & Nicotinamide phosphoribosyltransferase & Up \\
\hline NFIL3 & Nuclear factor, interleukin 3 regulated & Up \\
\hline NR4A2 & Nuclear receptor subfamily 4 group A member 2 & Up \\
\hline OSM & Oncostatin $\mathrm{M}$ & Up \\
\hline PFKFB3 & 6-phosphofructo-2-kinase/fructose-2,6-biphosphatase 3 & Up \\
\hline PLAUR & Plasminogen activator, urokinase receptor & Up \\
\hline PPP1R15A & Protein phosphatase 1 regulatory subunit $15 \mathrm{~A}$ & Up \\
\hline PTGS2 & Prostaglandin-endoperoxide synthase 2 & Up \\
\hline PTX3 & Pentraxin 3 & $\mathrm{Up}$ \\
\hline PVALB & Parvalbumin & Up \\
\hline RAB20 & RAB20, member RAS oncogene family & Up \\
\hline RGS1 & Regulator of $\mathrm{G}$ protein signaling 1 & Up \\
\hline SAMSN1 & SAM domain, SH3 domain and nuclear localization signals 1 & Up \\
\hline SGK1 & Serum/glucocorticoid regulated kinase 1 & Up \\
\hline STX11 & Syntaxin 11 & Up \\
\hline TNFRSF21 & TNF receptor superfamily member 21 & Up \\
\hline TRIB1 & Tribbles pseudokinase 1 & Up \\
\hline HIST2H2BE & H2B clustered histone $21 ?$ & Up \\
\hline LOC 100129518 & SOD2 overlapping transcript 1, SOD2 & Up \\
\hline ABHD17A & Abhydrolase domain containing 17A & Down \\
\hline IFT74 & Intraflagellar transport 74 & Down \\
\hline MPHOSPH8 & M-phase phosphoprotein 8 & Down \\
\hline RBM41 & RNA binding motif protein 41 & Down \\
\hline TIPRL & TOR signaling pathway regulator & Down \\
\hline NOTCH2NL & Notch 2 N-terminal like A & Down \\
\hline RP11-395B7.7 & Pre-mRNA processing factor 31 & Down \\
\hline
\end{tabular}


Table II. Gene Ontology analysis terms for the shared differentially expressed genes between the Crohn's disease and breast cancer datasets.

A, Biological process

\begin{tabular}{|c|c|c|c|}
\hline Term & Gene ID & Count & P-value \\
\hline GO:0060326-cell chemotaxis & CXCL2/HBEGF/CXCL8/IL1B/EGR3/C5AR1/CCR1 & 7 & $5.53 \times 10^{-06}$ \\
\hline $\begin{array}{l}\text { GO:0032103-positive regulation of } \\
\text { response to external stimulus }\end{array}$ & CXCL2/CXCL8/IL1B/PTGS2/C5AR1/CCR1/OSM & 7 & $7.19 \times 10^{-06}$ \\
\hline GO:0032496-response to lipopolysaccharide & $\begin{array}{l}\text { CXCL2/CXCL8/TRIB1/IL1B/ TNFRSF21/PTGS2/ } \\
\text { C5AR1 }\end{array}$ & 7 & $1.54 \times 10^{-05}$ \\
\hline
\end{tabular}

B, Molecular function

\begin{tabular}{|c|c|c|c|}
\hline Term & Gene ID & Count & P-value \\
\hline GO:0005125-cytokine activity & $\begin{array}{l}\text { CXCL2/CXCL8/IL1B/NAMPT/OSM/ } \\
\text { AREG }\end{array}$ & 6 & $2.09 \times 10^{-05}$ \\
\hline GO:0048018-receptor ligand activity & $\begin{array}{l}\text { CXCL2/HBEGF/CXCL8/IL1B/ } \\
\text { NAMPT/OSM/AREG }\end{array}$ & 7 & $1.58 \times 10^{-4}$ \\
\hline GO:0030545-receptor regulator activity & $\begin{array}{l}\text { CXCL2/HBEGF/CXCL8/IL1B/ } \\
\text { NAMPT/OSM/AREG }\end{array}$ & 7 & $2.31 \times 10^{-4}$ \\
\hline $\begin{array}{l}\text { GO:0008474-palmitoyl-(protein) hydrolase } \\
\text { activity }\end{array}$ & ABHD17A & 1 & $3.453 \times 10^{-3}$ \\
\hline GO:0098599-palmitoyl hydrolase activity & ABHD17A & 1 & $3.453 \times 10^{-3}$ \\
\hline GO:0016790-thiolester hydrolase activity & ABHD17A & 1 & $1.2042 \times 10^{-2}$ \\
\hline
\end{tabular}

Table III. Ten hub differentially expressed genes shared between the Crohn's disease and breast cancer datasets.

\begin{tabular}{llc}
\hline Gene & \multicolumn{1}{c}{ Gene name } & Degree \\
\hline CXCL8 & C-X-C motif chemokine ligand 8 & 14 \\
$I L 1 B$ & Interleukin $1 \beta$ & 13 \\
$E G R 1$ & Early growth response 1 & 10 \\
$P T G S 2$ & Prostaglandin-endoperoxide synthase 2 & 9 \\
$E G R 2$ & Early growth response 2 & 9 \\
FOSB & FosB proto-oncogene, AP-1 transcription factor subunit & 6 \\
LOC100129518 & SOD2 overlapping transcript 1, SOD2 & 5 \\
HBEGF & Heparin binding EGF like growth factor & 5 \\
$P L A U R$ & C-X-C motif chemokine ligand 2 & 5 \\
\hline
\end{tabular}

in the NF- $\mathrm{NB}$ signaling pathway. The inflammatory genes CXCL8 (encoding the IL- 8 protein), IL1 $\beta$ and PTGS2 were mainly involved in cell survival, positive/negative feedback loops of inflammation processes in different types of disease, such as colorectal cancer (17) and rheumatoid arthritis (18). In Fig. 5, the expression of the inflammatory genes IL1 $\beta$, PTGS2 and E3 SUMO-protein ligase EGR2/3 were associated with T helper (Th)17 cell differentiation function. In Fig. 6, IL-17B/IL-17RB activated IL1 $\beta$ through AP1 complex activation, which leads to inflammation. A comprehensive pathway and network analysis of hub genes were identified.

\section{Discussion}

Patients with IBD have a greater risk of developing cancer compared with patients without IBD (2). A previous study showed that patients with $\mathrm{CD}$ have a high risk of developing colorectal cancer (19). Patients with CD also have a higher risk of developing breast cancer than those without CD (2). Based on these observations, new treatment strategies need to be developed to treat patients with IBD and breast cancer. The molecular mechanisms underlying the association between $\mathrm{CD}$ and breast cancer may provide important information to 
A

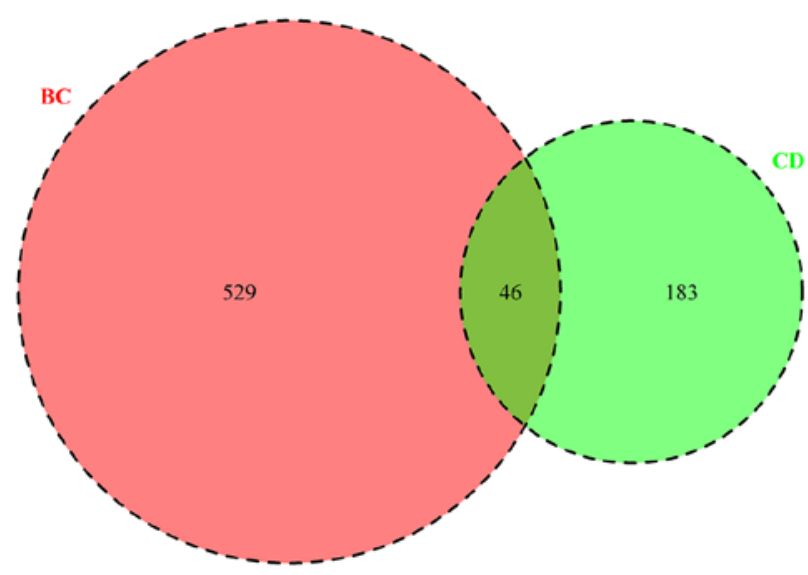

B

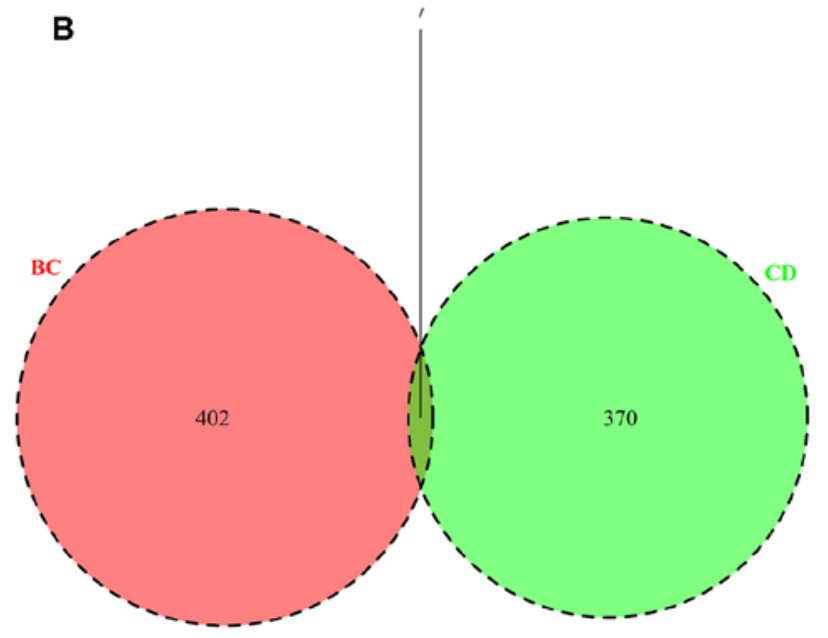

Figure 1. Overlap of the DEGs from the GSE3365 (CD; green) and GSE27562 (BC; red) datasets. (A) Upregulated DEGs and (B) downregulated DEGs. DEGs, differentially expressed genes; $\mathrm{CD}$, Crohn's disease; $\mathrm{BC}$, breast cancer; p.adjust, adjusted P-value, which refers to the adjustment of a p-value in a single significance test when multiple comparisons/testing need to be performed.

A

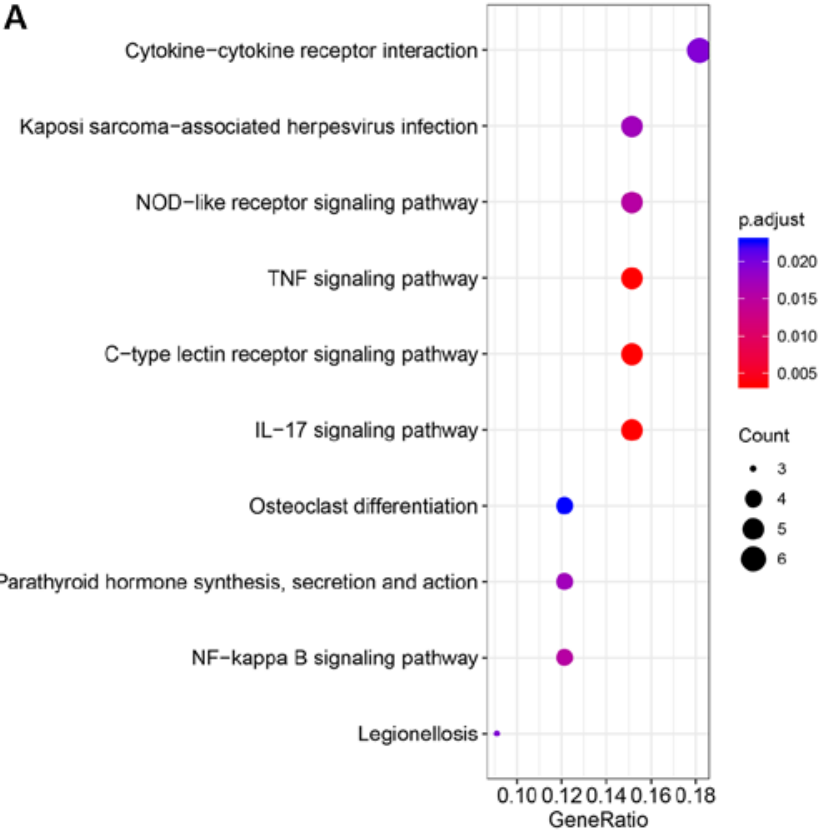

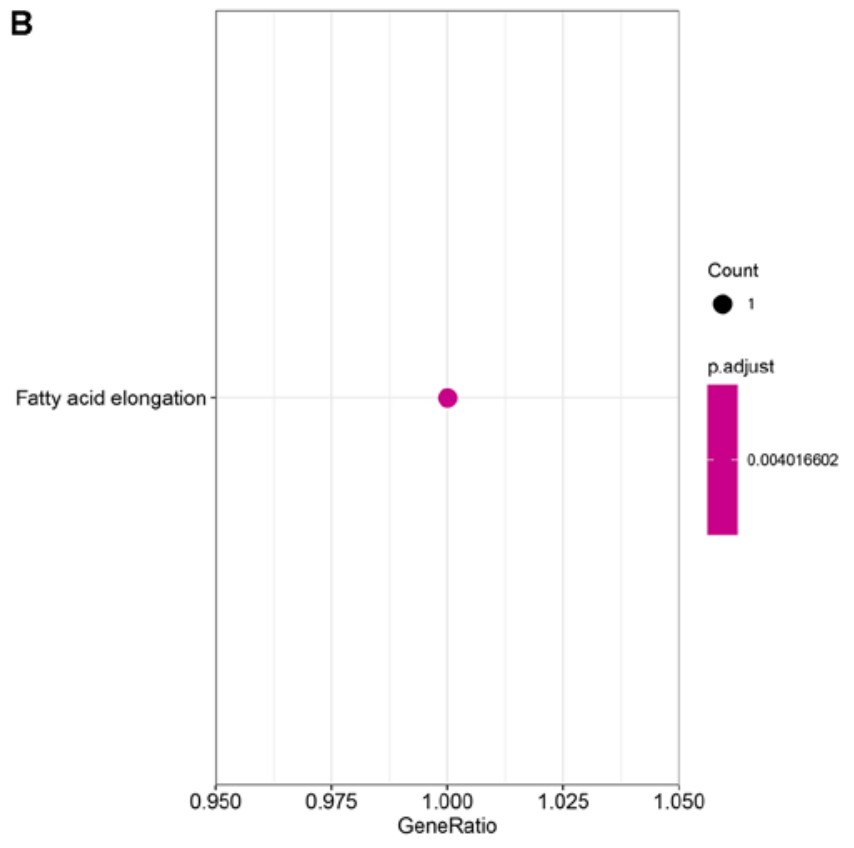

B

Figure 2. The Kyoto Encyclopedia of Genes and Genomes enrichment analyses of the overlapping DEGs from the GSE3365 (Crohn's disease) and GSE27562 (breast cancer) datasets. (A) Upregulated DEGs and (B) downregulated DEGs. DEGs, differentially expressed genes; TNF, tumor necrosis factor; IL, interleukin; p-adjust, adjusted P-value.

inform such therapy. The present study identified 53 overlapping DEGs between the $\mathrm{CD}$ and $\mathrm{BC}$ group. A gene interaction network based on the overlapping genes was constructed, which demonstrated how the molecular mechanisms underpinning $\mathrm{CD}$ are associated with breast cancer.

GO term annotation and KEGG pathway enrichment analyses were then performed. The results showed that the upregulated overlapping DEGs are annotated with 'cell chemotaxis'. This suggested that they may be mainly involved in inflammatory pathways. Moreover, the KEGG analysis showed that the upregulated overlapping DEGs in the $\mathrm{CD}$ vs. $\mathrm{BC}$ groups were enriched in the NF- $\mathrm{KB}$ signaling pathway. These findings are consistent with the results of previous studies. According to a previous study, the NF- $\kappa B$ signaling pathway demonstrates unregulated activation and plays a key role in the pathogenesis of Crohn's disease (20). Interestingly, in the peripheral blood of patients with breast cancer, it was reported that certain genes were enriched in the GO term ' $\mathrm{NF}-\kappa \mathrm{B}$ ' and some upregulated genes were involved in the canonical mitogen-activated protein kinase signaling pathway, which is associated with NF- $\kappa \mathrm{B}$ pathway signaling (7). This consistency implies that similar molecular mechanisms are shared between $\mathrm{CD}$ and breast cancer pathogenesis. 


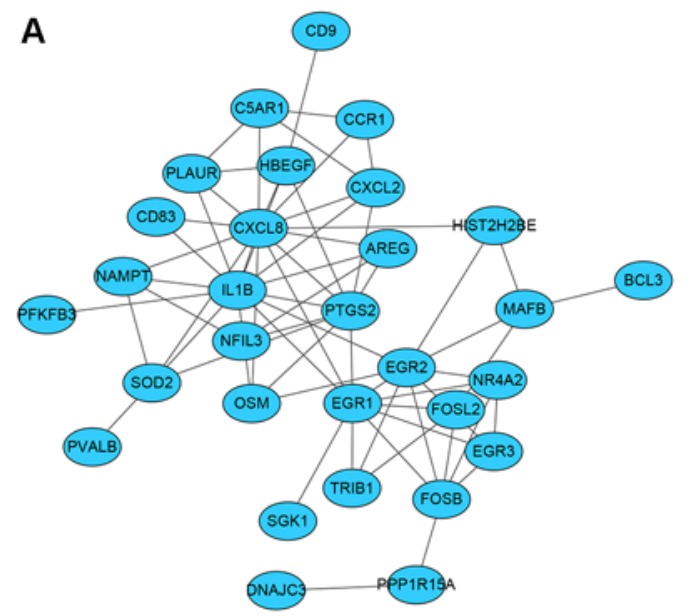

B

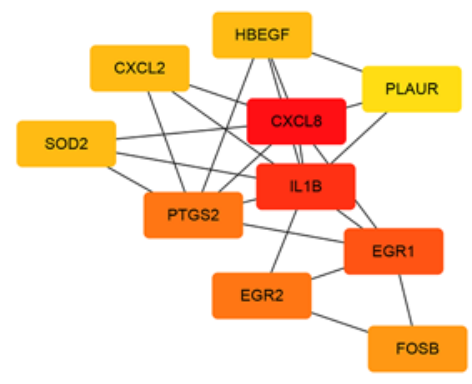

C

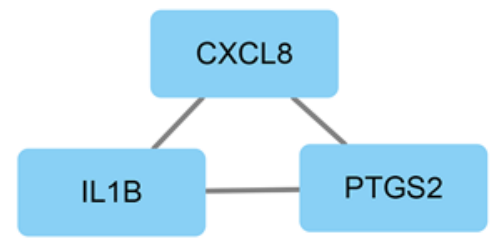

Figure 3. Gene interaction network and the significant module in Crohn's disease vs. breast cancer. (A) Gene interaction network of the DEGs. (B) Hub gene network in the gene interaction network. (C) The significant module of the gene interaction network. All nodes represent upregulated genes. DNAJC3, DnaJ heat shock protein family (Hsp40) member C3; CXCL1, C-X-C motif chemokine ligand 1; CXCL2, C-X-C motif chemokine ligand 2; PLAUR, plasminogen activator, urokinase receptor; SGK1, serum/glucocorticoid regulated kinase 1; FOSL2, FOS-like 2; HBEGF, heparin-binding EGF-like growth factor; NR4A2, nuclear receptor subfamily 4 group A member 2; MAFB, MAF bZIP transcription factor B; CXCL8, C-X-C motif chemokine ligand 8; PFKFB3, 6-phosphofructo-2-kinase/fructose-2,6-biphosphatase 3; TRIB1, tribbles pseudokinase 1; IL1B, interleukin $1 \beta$; NFIL3, nuclear factor, interleukin 3-regulated; EGR3, early growth response 3; EGR1, early growth response 1; PTGS2, prostaglandin-endoperoxide synthase 2; EGR2, early growth response 2; BCL3, BCL3 transcription coactivator; HIST2H2BE, histone cluster $2 \mathrm{H} 2 \mathrm{~B}$ family member e; PPP1R15A, protein phosphatase 1 regulatory subunit 15A; C5AR1,complement C5a receptor 1; CCR1, C-C motif chemokine receptor 1; CD9, cluster of differentiation 9; FOSB, FosB proto-oncogene, AP-1 transcription factor subunit; NAMPT, nicotinamide phosphoribosyltransferase; PVALB, parvalbumin; OSM, oncostatin M; CD83, cluster of differentiation 83; AREG, amphiregulin; SOD2, superoxide dismutase 2.

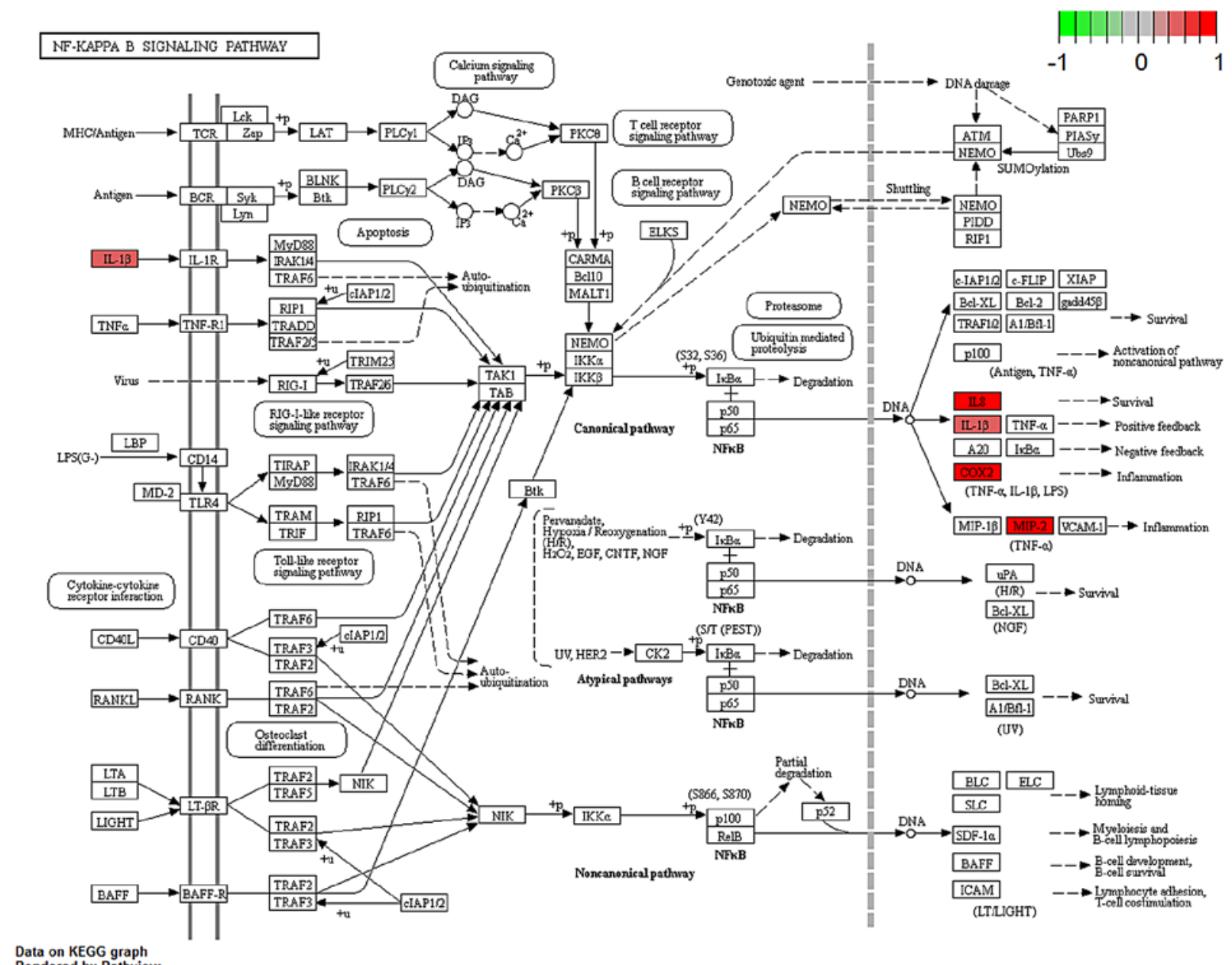

Figure 4. Gene expression profiles of the NF-kB signaling pathway in breast cancer. The red and green colors mark the $\log _{2}$ (fold-change) of the corresponding genes. IL1B, interleukin $1 \beta$; IL8, interleukin 8; COX2 (PTGS2), prostaglandin-endoperoxide synthase 2; MIP-2, macrophage inflammatory protein 2. 


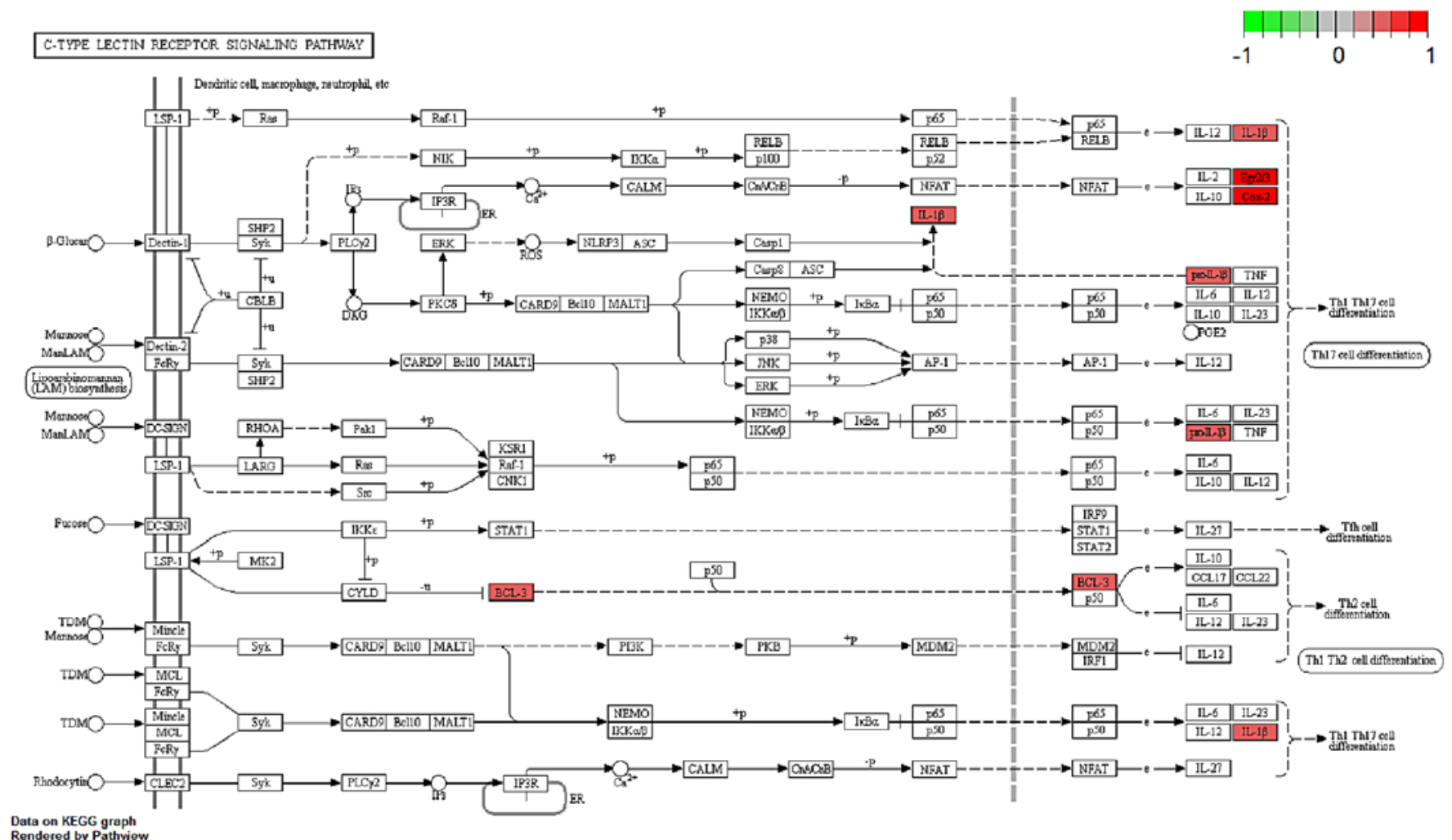

Data on KEGG graph
Rendered by Pathwiow

Figure 5. Gene expression profiles of the C-type lectin receptor signaling pathway in the breast cancer group. The red and green colors mark the $\log _{2}$ (fold-change) of the corresponding genes. BCL3, BCL3 transcription coactivator; proIL- $\beta$, interleukin-1 $\beta$ precursor; IL-1 $\beta$, interleukin $1 \beta$.

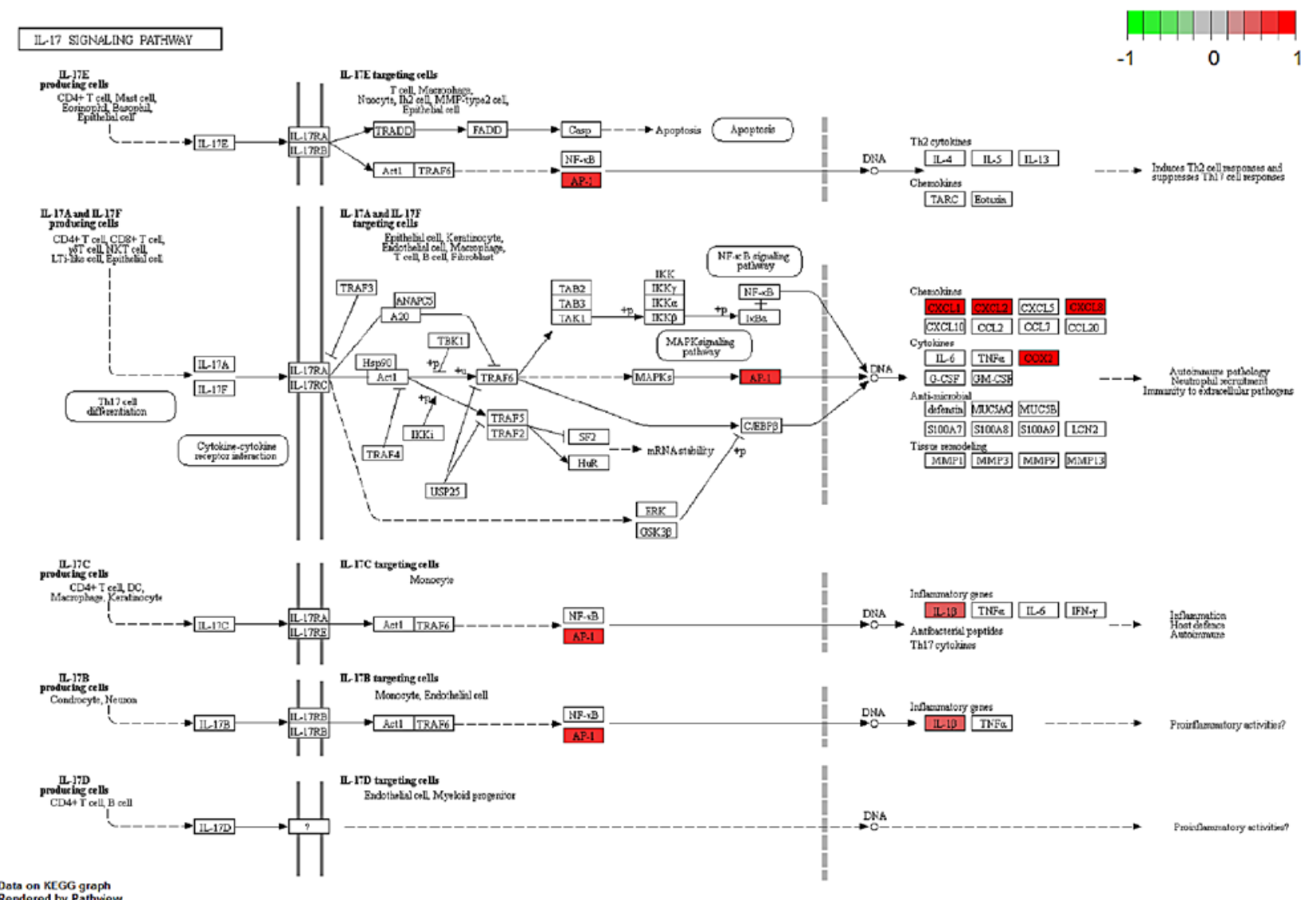

Figure 6. Gene expression profiles of the IL-17 signaling pathway in the breast cancer group. The red and green colors mark the $\log _{2}$ (fold-change) of the corresponding genes. COX2 (PTGS2), prostaglandin-endoperoxide synthase; IL-1 $\beta$, interleukin $1 \beta$; AP-1, activator protein-1; CXCL1, C-X-C motif chemokine ligand 1; CXCL2, C-X-C motif chemokine ligand 2; CXCL8, C-X-C motif chemokine ligand 8. 
The present study presented the transcriptional regulation associations of the hub DEGs in the NF- $\kappa \mathrm{B}$ and IL-17 signaling pathways in patients with $\mathrm{CD}$ and breast cancer. In the colon of patients with $\mathrm{CD}$, the predominance of Th1/Th17 cells lead to the upregulation of multiple cytokines, including TNF- $\alpha$, IFN- $\gamma$, IL-1, IL-12 and IL-17. In mononuclear and epithelial cells of the inflamed colon, inflammatory cytokines, such as TNF- $\alpha$, may activate the NF- $\kappa \mathrm{B}$ transcription factor to induce expression of the general inflammatory gene $(21,22)$. Numerous blood Th17 cells infiltrate the inflamed mucosa of patients with Crohn's Disease (23). When compared with controls, the peripheral CD4+ T cells of patients with $C D$ show increased bacterial responses due to overexpression of Th17-associated genes, including $I L-17 A, I L 17 F$, nuclear receptor ROR- $\gamma$, C-C chemokine receptor type 6 and C-C motif chemokine 20 (24). Moreover, the peripheral blood monocytes of patients with $\mathrm{CD}$ express higher levels of IL1 $\beta$, CCL2 and CCL5 (25). It is well known that IL1 $\beta$ is a factor that promotes the expansion of Th17 cells (26). Furthermore, when the circulating Th17 cells of patients with CD reach breast tumor tissue, Th17 cell activation may be exacerbated (26). Breast tumor cells can interact with activated $\mathrm{T}$ cells via CD40-CD40L, by which TGF $\beta$ production and the differentiation of Th17 cells increases (27). In the end, inflammatory blood Th17 cells, together with $\mathrm{NF}-\kappa \mathrm{B}$ signaling, may serve a role in linking inflammation, immunity and breast oncogenesis (28). In fact, Th17 cells infiltrate breast cancer tissues, including the inflammatory tumor microenvironment. For example, IL-17 can upregulate the expression of CXCL1 on breast cancer cells, which can activate the $\mathrm{AKT} / \mathrm{NF}-\kappa \mathrm{B}$ signaling pathway to enhance breast cancer growth and metastasis (29). Th17-associated inflammation may also lead to the upregulation of IL17B, another member of the IL-17 cytokine family, and its receptor (IL17RB) in breast epithelial malignant cells, which can induce the activation of the ERK1/2, NF- $\mathrm{B}$ and Bcl-2 pathways and enhance the inflammation and development of breast cancer (30). In addition, tumor-associated monocytes and macrophages can create a cancer stem cell (CSC) niche through juxtacrine signaling, including NF- $\kappa \mathrm{B}$ (31). The present hub DEGs in the gene interaction networks may participate in this process. In the CSC niche, the NF- $\kappa \mathrm{B}$ may translocate into the nucleus and subsequently activate the expression of inflammatory genes CXCL8, IL1 $\beta$ and PTGS2 (32,33).

Hub genes in significant modules overexpressed by peripheral blood cells that are involved in the mentioned pathways may contribute to development of breast cancer in CD. These inflammatory mediators are CXCL8, IL1 $\beta$ and PTGS2. CXCL8, also known as IL-8, may serve an important role in cancer progression by regulating CSC proliferation and self-renewal (34). In addition, CXCL8 also has a role in neovascularization, which contributes to tumor growth and metastasis in the tumor microenvironment (35). IL-1 $\beta$ is another proinflammatory mediator that can enhance tumor-promoting inflammation in breast cancer (36). IL-1 $\beta$ can activate the $\beta$-catenin signaling pathway to induce the epithelial-mesenchymal transition (EMT) in breast cancer cells (37). IL-1 $\beta$ induces the migration of breast cancer cells through the induction of hypoxia-inducible factor (HIF)-1 $\alpha$ (38). However, inhibition of HIF- $1 \alpha$ cannot prevent the breast cancer cell migration activated by IL-1 $\beta$ under hypoxic conditions, in which $\mathrm{NF \kappa B} / \mathrm{CXCL8}$ signaling may play a compensatory role (39). PTGS2, formerly known as cyclooxygenase 2 (COX-2), catalyzes the production of inflammatory prostaglandins that exacerbate inflammation (40). PTGS2-induced PGE2 production promotes the migration and EMT of human breast cancer cells (41). The inhibition of the PTGS2 signaling pathway can inhibit the growth of inflammatory breast cancer in vivo (42). PTGS2 plays a role in HIF1 activation and promotes tumor angiogenesis in breast carcinoma (43). PTGS2 can also interact with other crucial genes, such as IL8, IL1 $\beta$ and CXCL2 in tumor-associated inflammation. Expression of IL8/CXCL8 increases in PTGS2 overexpressed NSCLC cell lines (44). Taken together, the results of the present study demonstrated that a series of inflammatory proteins (such as CXCL8, IL1 $\beta$ and PTGS2) overexpressed by peripheral blood cells may increase systemic inflammation in female patients with CD (45). Systemic inflammation can affect tumorigenesis and progression in breast cancer by establishing a tumor immune microenvironment (TIME) (46).

The limitations of the present study include the existence of batch effect, which refers to non-biological variation between measurements of different groups of samples, and the lack of samples from patients with breast cancer with comorbid CD, thus indicating that present data may be insufficient to highlight the molecular basis underlying the genetic interaction between these two diseases. To overcome these limitations, further studies should use microarray datasets in which batch effects are removed, and focus on investigating individuals who have both $\mathrm{CD}$ and breast cancer.

In summary, a total of 53 overlapping DEGs were identified between Crohn's disease and breast cancer. Functional analyses of the upregulated overlapping DEGs showed that the two diseases are associated with the IL-17 and NF- $\kappa$ B signaling pathways. Gene interaction network and module analysis demonstrated that the major hub genes were CXCL8, IL1 $\beta$ and PTGS2. The genes and pathways identified in the present study may help inform our understanding of the possible mechanisms underlying why Crohn's disease can be a risk factor for developing breast cancer.

\section{Acknowledgements}

Not applicable.

\section{Funding}

The present study was supported by the National Natural Science Foundation of China (NSFC; grant nos. 81970457, 91842302, 31470876 and 91629102).

\section{Availability of data and materials}

All datasets for this study (GSE3365 and GSE27562) are included in the GEO program (https://www.ncbi.nlm.nih.gov/geo).

\section{Authors' contributions}

RY and JZ designed the study. JZ acquired, analyzed and interpreted the data. RY and JZ wrote the paper. All authors read and approved the final manuscript. 


\section{Ethics approval and consent to participate}

Not applicable.

\section{Patient consent for publication}

Not applicable.

\section{Competing interests}

The authors declare that they have no competing interests.

\section{References}

1. Weimers $P$ and Munkholm P: The natural history of IBD: Lessons learned. Curr Treat Options Gastroenterol 16: 101-111, 2018.

2. Hovde Ø, Høivik ML, Henriksen M, Solberg IC, Småstuen MC and Moum BA: Malignancies in patients with inflammatory bowel disease: Results from 20 Years of follow-up in the IBSEN Study. J Crohns Colitis 11: 571-577, 2017.

3. Pellino G, Sciaudone G, Patturelli M, Candilio G, De Fatico GS Landino I, Facchiano A, Vastarella A, Canonico S, Riegler G and Selvaggi F: Relatives of Crohn's disease patients and breast cancer: An overlooked condition. Int J Surg 12 (Suppl 1): S156-S158, 2014

4. Riegler G, Caserta L, Castiglione F, Esposito I, Valpiani D, Annese V, Zoli G, Gionchetti P, Viscido A, Sturniolo GC, et al: Increased risk of breast cancer in first-degree relatives of Crohn's disease patients. An IG-IBD study. Dig Liver Dis 38: 18-23, 2006.

5. Barrett T, Wilhite SE, Ledoux P, Evangelista C, Kim IF Tomashevsky M, Marshall KA, Phillippy KH, Sherman PM, Holko M, et al: NCBI GEO: Archive for functional genomics data sets-update. Nucleic Acids Res 41 (Database Issue): D991-D995, 2013.

6. Burczynski ME, Peterson RL, Twine NC, Zuberek KA, Brodeur BJ, Casciotti L, Maganti V, Reddy PS, Strahs A, Immermann F, et al: Molecular classification of Crohn's disease and ulcerative colitis patients using transcriptional profiles in peripheral blood mononuclear cells. J Mol Diagn 8: 51-61, 2006.

7. Labreche HG, Nevins JR and Huang E: Integrating factor analysis and a transgenic mouse model to reveal a peripheral blood predictor of breast tumors. BMC Med Genomics 4: 61, 2011.

8. Lenz M, Müller FJ, Zenke M and Schuppert A: Principal components analysis and the reported low intrinsic dimensionality of gene expression microarray data. Sci Rep 6: 25696, 2016

9. Yu G, Wang LG, Han Y and He QY: ClusterProfiler: An R package for comparing biological themes among gene clusters. OMICS 16: 284-287, 2012

10. Shannon P, Markiel A, Ozier O, Baliga NS, Wang JT, Ramage D, Amin N, Schwikowski B and Ideker T: Cytoscape: A software environment for integrated models of biomolecular interaction networks. Genome Res 13: 2498-504, 2003.

11. Luo W and Brouwer C: Pathview: An R/Bioconductor package for pathway-based data integration and visualization. Bioinformatics 29: 1830-1831, 2013.

12. Welte $T$ and Zhang XHF: Interleukin-17 could promote breast cancer progression at several stages of the disease. Mediators Inflamm 2015: 804347, 2015

13. Wolfkamp SC, Verstege MI, Vogels EW, Meisner S, Verseijden C Stokkers PC and te Velde AA: Single nucleotide polymorphisms in C-type lectin genes, clustered in the IBD2 and IBD6 susceptibility loci, may play a role in the pathogenesis of inflammatory bowel diseases. Eur J Gastroenterol Hepatol 24: 965-970, 2012.

14. Hohenberger M, Cardwell LA, Oussedik E and Feldman SR: Interleukin-17 inhibition: Role in psoriasis and inflammatory bowel disease. J Dermatolog Treat 29: 13-18, 2018.

15. Tambuwala MM: Natural nuclear factor kappa beta inhibitors: Safe therapeutic options for inflammatory bowel disease. Inflamm Bowel Dis 22: 719-23, 2016.

16. Wang W, Nag SA and Zhang R: Targeting the NFאB signaling pathways for breast cancer prevention and therapy. Curr Med Chem 22: 264-89, 2015

17. Abdulamir AS, Hafidh RR and Bakar FA: Molecular detection, quantification, and isolation of Streptococcus gallolyticus bacteria colonizing colorectal tumors: Inflammation-driven potential of carcinogenesis via IL-1, COX-2, and IL-8. Mol Cancer 9: 249, 2010.
18. Lee YA, Choi HM, Lee SH, Yang HI, Yoo MC, Hong SJ and Kim KS: Synergy between adiponectin and interleukin-1 $\beta$ on the expression of interleukin-6, interleukin-8, and cyclooxygenase-2 in fibroblast-like synoviocytes. Exp Mol Med 44: 440-447, 2012.

19. Kameyama $H$, Nagahashi $M$, Shimada $Y$, Tajima $Y$, Ichikawa $H$, Nakano M, Sakata J, Kobayashi T, Narayanan S, Takabe K and Wakai T: Genomic characterization of colitis-associated colorectal cancer. World J Surg Oncol 16: 121, 2018.

20. Han YM, Koh J, Kim JW, Lee C, Koh SJ, Kim B, Lee KL, Im JP and Kim JS: NF-kappa B activation correlates with disease phenotype in Crohn's disease. PLoS One 12: e0182071, 2017.

21. Kmieć Z, Cyman M and Ślebioda TJ: Cells of the innate and adaptive immunity and their interactions in inflammatory bowel disease. Adv Med Sci 62: 1-16, 2017.

22. Schreiber S, Nikolaus S and Hampe J: Activation of nuclear factor $\kappa \mathrm{B}$ in inflammatory bowel disease. Gut 42: 477-484, 1998.

23. Gálvez J: Role of Th17 cells in the pathogenesis of human IBD. ISRN Inflamm 2014: 928461, 2014.

24. Bassolas-Molina H, Raymond E, Labadia M, Labadia M, Wahle J, Ferrer-Picón E, Panzenbeck M, Zheng J, Harcken C, Hughes R, et al: An ROR $\gamma$ oral inhibitor modulates IL-17 responses in peripheral blood and intestinal Mucosa of Crohn's disease patients. Front Immunol 9: 2307, 2018.

25. Schwarzmaier D, Foell D, Weinhage T, Varga G and Dabritz J: Peripheral monocyte functions and activation in patients with quiescent Crohn's disease. PLoS One 8: e62761, 2013.

26. Brand S: Crohn's disease: Th1, Th17 or Both? The change of a paradigm: New immunological and genetic insights implicate Th17 cells in the pathogenesis of Crohn's disease. Gut 58: 1152-1167, 2009.

27. Kim H, Kim Y, Bae S, Kong JM, Choi J, Jang M, Choi J, Hong JM, Hwang YI, Kang JS and Lee WJ: Direct interaction of CD40 on tumor cells with CD40L on T cells increases the proliferation of tumor cells by enhancing TGF- $\beta$ production and Th17 differentiation. PLoS One 10: e0125742, 2015.

28. Wang J, Cai D, Ma B, Wu G and Wu J: Skewing the balance of regulatory T-cells and T-helper 17 cells in breast cancer patients. J Int Med Res 39: 691-701, 2011.

29. Ma K, Yang L, Shen R, Kong B, Chen W, Liang J, Tang G and Zhang B: Th17 cells regulate the production of CXCL1 in breast cancer. Int Immunopharmacol 56: 320-329, 2018.

30. Alinejad V, Dolati S, Motallebnezhad M and Yousefi M: The role of IL17B-IL17RB signaling pathway in breast cancer. Biomed Pharmacother 88: 795-803, 2017.

31. Lu H, Clauser KR, Tam WL, Fröse J, Ye X, Eaton EN, Reinhardt F, Donnenberg VS, Bhargava R, Carr SA and Weinberg RA: A breast cancer stem cell niche supported by juxtacrine signalling from monocytes and macrophages. Nat Cell Biol 16: 1105-1117, 2014.

32. Li B, Lu Y, Yu L, Han X, Wang H, Mao J, Shen J, Wang B, Tang J, Li C and Song B: miR-221/222 promote cancer stem-like cell properties and tumor growth of breast cancer via targeting PTEN and sustained Akt/NF-кB/COX-2 activation. Chem Biol Interact 277: 33-42, 2017.

33. Nomura A, Gupta VK, Dauer P, Sharma NS, Dudeja V, Merchant N, Saluja AK and Banerjee S: NFאB-mediated invasiveness in $\mathrm{CD}_{133^{+}}$pancreatic TICs is regulated by autocrine and paracrine activation of IL1 signaling. Mol Cancer Res 16: 162-172, 2018.

34. Ha H, Debnath B and Neamati N: Role of the CXCL8-CXCR1/2 axis in cancer and inflammatory diseases. Theranostics 7 : 1543-1588, 2017.

35. Liu Q, Li A, Tian Y, Wu JD, Liu Y, Li T, Chen Y, Han X and Wu K: The CXCL8-CXCR1/2 pathways in cancer. Cytokine Growth Factor Rev 31: 61-71, 2016.

36. Dinarello CA: An interleukin-1 signature in breast cancer treated with interleukin-1 receptor blockade: Implications for treating cytokine release syndrome of checkpoint inhibitors. Cancer Res 78: 5200-5202, 2018.

37. Perez-Yepez EA, Ayala-Sumuano JT, Lezama R and Meza I: A novel $\beta$-catenin signaling pathway activated by IL-1 $1 \beta$ leads to the onset of epithelial-mesenchymal transition in breast cancer cells. Cancer Lett 354: 164-171, 2014

38. Naldini A, Filippi I, Miglietta D, Moschetta M, Giavazzi R and Carraro F: Interleukin-1 $\beta$ regulates the migratory potential of MDAMB231 breast cancer cells through the hypoxia-inducible factor-1 $\alpha$. Eur J Cancer 46: 3400-3408, 2010.

39. Filippi I, Carraro F and Naldini A: Interleukin-1 $\beta$ affects MDAMB231 breast cancer cell migration under hypoxia: Role of HIF-1 $\alpha$ and NFאB transcription factors. Mediators Inflamm 2015: 789414, 2015

40. O'Connor PM, Lapointe TK, Beck PL and Buret AG: Mechanisms by which inflammation may increase intestinal cancer risk in inflammatory bowel disease. Inflamm Bowel Dis 16: 1411-1420, 2010. 
41. Reader J, Holt D and Fulton A: Prostaglandin E2 EP receptors as therapeutic targets in breast cancer. Cancer Metastasis Rev 30: 449-463, 2011.

42. Wang X, Reyes ME, Zhang D, Funakoshi Y, Trape AP, Gong Y, Kogawa T, Eckhardt BL, Masuda H, Pirman DA Jr, et al: EGFR signaling promotes inflammation and cancer stem-like activity in inflammatory breast cancer. Oncotarget 8: 67904-67917, 2017.

43. Maroni P, Bendinelli P, Matteucci E and Desiderio MA: The therapeutic effect of miR-125b is enhanced by the prostaglandin endoperoxide synthase $2 /$ cyclooxygenase 2 blockade and hampers ETS1 in the context of the microenvironment of bone metastasis. Cell Death Dis 9: 472, 2018.

44. Põld M,Zhu LX,SharmaS,Burdick MD,Lin Y,Lee PP,Põld A,LuoJ, KrysanK,DohadwalaM,etal:Cyclooxygenase-2-dependentexpression of angiogenic CXC chemokines ENA-78/CXC Ligand (CXCL) 5 and interleukin-8/CXCL8 in human non-small cell lung cancer. Cancer Res 64: 1853-1860, 2004.
45. Gross V, Andus T, Leser HG, Roth $M$ and Schölmerich J: Inflammatory mediators in chronic inflammatory bowel diseases. Klin Wochenschr 69: 981-987, 1991.

46. Deshmukh SK, Srivastava SK, Poosarla T, Dyess DL, Holliday NP, Singh AP and Singh S: Inflammation, immunosuppressive microenvironment and breast cancer: Opportunities for cancer prevention and therapy. Ann Transl Med 7: 593, 2019.

This work is licensed under a Creative Commons Attribution-NonCommercial-NoDerivatives 4.0 International (CC BY-NC-ND 4.0) License. 